\title{
Multiple sgRNAs facilitate base editing- mediated i-stop to induce complete and precise gene disruption
}

\section{Dear Editor,}

Gene editing is a process to introduce desired changes into targeted loci of genomic DNA. Recently, type II clustered regularly interspaced short palindromic repeats-associated Cas9 endonuclease (CRISPR/Cas9) system has been demonstrated as a versatile tool for engineering eukaryote genome (Hsu et al., 2014), such as in mice (Zuo et al., 2017). CRISPR/Cas9-mediated genome editing is achieved by the error-prone DNA repair of non-homologous end joining (NHEJ) after double strand DNA cleavage. However, the editing results are unreliable due to uncontrolled random indels. Moreover, it was also occasionally reported that Cas9 may induce troublesome off-target effects (Hsu et al., 2013; Pattanayak et al., 2013; Cho et al., 2014).

Scientists are making continuous efforts to modify and optimize the gene editing tools. In a landmark study, Komor et al. developed a 'DNA' base editor (BE), a novel genome editing tool which is applicable to change $\mathrm{C} / \mathrm{G}$ base pairs to A/T without introducing DNA double strand breaks. Thereafter, various modifications have been created to the base editor system to improve its editing efficiency. BE3 can introduce C-to-T nucleotide substitution at the window of position 4-8 bases of the non-binding strand of the sgRNA (Komor et al., 2016). BE4max increased efficiency in a variety of mammalian cell types (Koblan et al., 2018). Interestingly, BE3 has been used to introduce early stop codon (TGA, TAG, TAA) from codons (CAA, CAG, CGA, TGG) to terminate gene expression (Kuscu et al., 2017), and provides a safer and much more precise knockout strategy than Cas9-mediated NHEJ (Kim et al., 2017; Komor et al., 2017).

In this study, we used BE3 and BE4max to edit mouse genome by introducing stop codon (i-stop) in coding region of specific genes. We tested a multiple sgRNAs strategy and the results indicated that multiple sgRNAs dramatically increase the efficiencies of BE3-mediated and BE4maxmediated editing in mouse embryos and successfully generated DKO (double knockout) mice by BE3-mediated i-stop targeting Tyr and Pdcd1.

First, mouse-derived Neuro-2a (N2a) cells were used as testing system. We designed and screened 14 sgRNAs targeting Tyr and Pdcd1 (7 sgRNAs for each gene), respectively. TYR gene encodes the tyrosinase enzyme, and its mutations result in impaired tyrosinase production leading to albinism (Witkop, 1979). PDCD1 is an immune checkpoint gene which guards against autoimmunity and regulatory $T$ cells (Fife and Pauken, 2011).

To screen the candidate sgRNA, BE3 plasmid and individual sgRNA were co-transfected into N2a cells. The results of chromatograms showed, 3 out of 7 sgRNAs for Tyr (Tyrsg1, Tyr-sg2 and Tyr-sg7 targeting exon1) and 3 out of 7 sgRNAs for Pdcd1 (Pdcd1-sg1, Pdcd1-sg2 and Pdcd1-sg3, targeting exon 1 , exon 2 and exon 3 , respectively) worked well in modifying the genome in coding regions (Fig. S1). We then test the editing efficiency of BE4max with these six sgRNAs (Fig. S3A). As expected, subsequent TA clone sequencing confirmed that all six sgRNAs introduced stop codon at the predicted sites with BE3 (Fig. S1D) or BE4max (Fig. S3B). For BE3, Tyr-sg1 and Tyr-sg2, Tyr-sg7 generated stop codon (Q48stop, W272stop, W12stop) at the frequencies of $13.3 \%, 22.2 \%$ and $14.3 \%$ respectively. On the other hand, Pdcd1-sg1, Pdcd1-sg2 and Pdcd1-sg3 generated stop codon (Q79stop, Q167stop and W12stop) at the frequencies of $10 \%, 37.5 \%$ and $20 \%$. For BE4max, Tyr-sg1, Tyr-sg2 and Tyr-sg7 generated stop codon at the frequencies of $50 \%$, $33.3 \%$ and $33.3 \%$, respectively, while Pdcd1-sg1, Pdcd1sg2 and Pdcd1-sg3 generated stop codon at the frequencies of $30 \%, 50 \%$ and $33.2 \%$. Thus, Tyr-sg1, 2, 7 and Pdcd1-sg1, 2, 3 were selected for the further study.

We then attempted to test the efficiency of i-stop conversion in mouse embryos. To test multiple sgRNAs strategies, different combinations of sgRNAs and BE mRNA or BE4max mRNA were co-injected into zygotes (50 ng/ $\mu \mathrm{L} \mathrm{BE}$ mRNA and $25 \mathrm{ng} / \mu \mathrm{L}$ sgRNAs) (Fig. 1B).

We successfully introduced stop codon (i-stop) with BE3. For Tyr, 2 out of 10 (20\%) blastocysts (\#5 and \#9) for Tyr-sg1 harbored genomic modification of synonymous mutation (G47G), while 5 out of $8(62.5 \%$ ) blastocysts (\#1-3, \#6 and \#7) harbored i-stop mutation for Tyr-sg1 combined with Tyrsg2, indicating that multiple sgRNAs can enhance i-stop introduction in embryos (Figs. 1C and S4A). For Pdcd1, 2 out of $10(20 \%)$ (\#5 and \#6), 2 out of $8(25 \%)$ (\#1 and \#7) and 5 

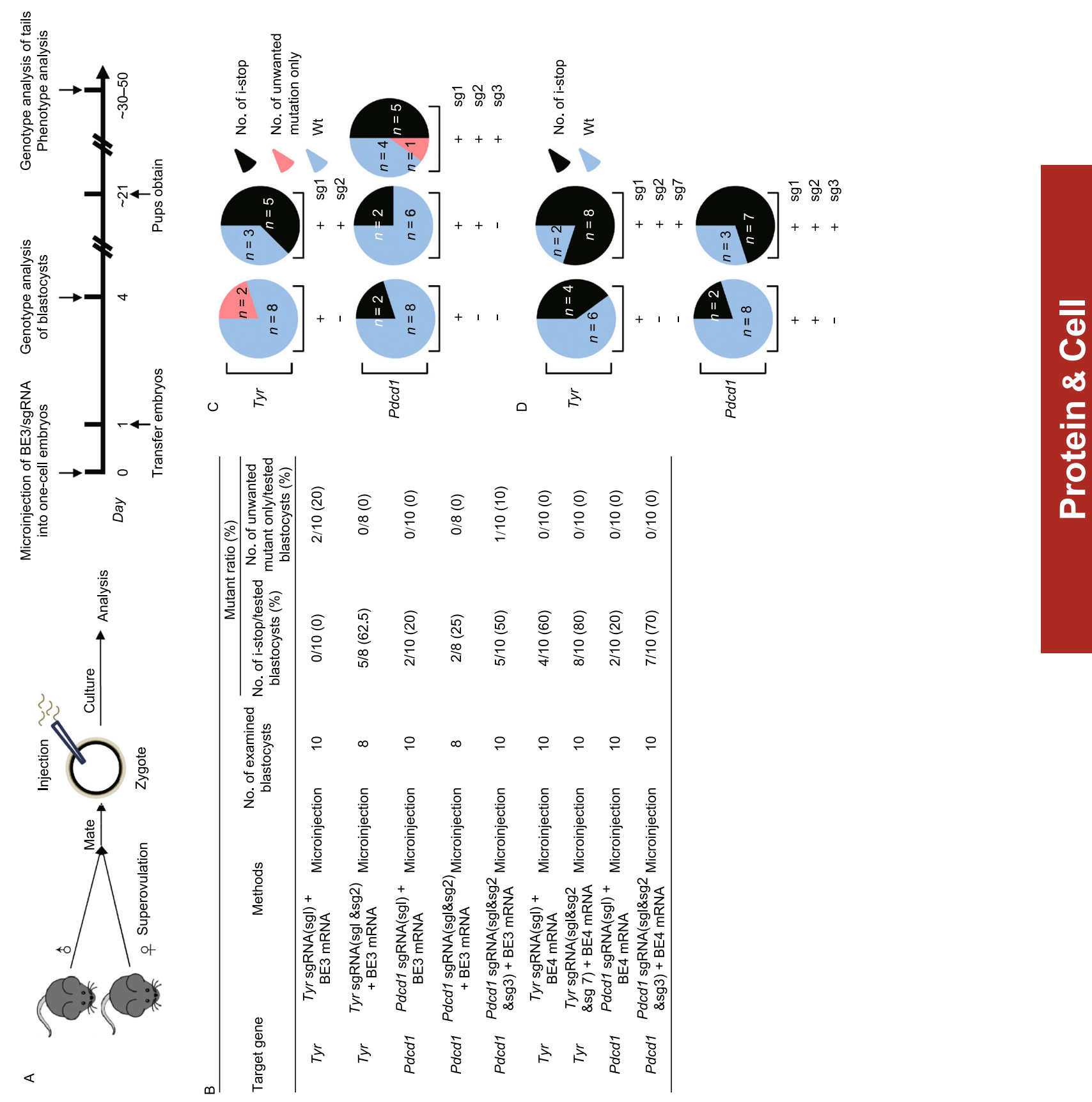
4 Figure 1. Efficient C-to-T substitution at Tyr and Pdcd1 loci in mouse embryos and mutant mice. (A) Representative schematic and timeline of experimental design. After mating and superovulation of mice, sgRNA and BE3 mRNA or BE4max mRNA were co-injected into one-cell embryos, then editing efficiency were detected at blastocyst stage and founder mice. (B) Summary of embryo manipulation. (C) The percentage of different mutation types in mouse embryos by BE3-mediated base editing. Black represents percentage of blastocysts harboring generated stop codons; Red represents percentage of blastocysts harboring unwanted mutations only; Blue represents percentage of Wt (wild type) blastocysts. The number was indicated on the chart. (D) The percentage of different mutation types in mouse embryos by BE4max-mediated base editing. Black represents percentage of blastocysts harboring generated stop codons; Blue represents percentage of Wt (wild type) blastocysts. The number was indicated on the chart. (E) Tyr mutant newborn pups that developed after co-injecting the BE3 mRNA and sgRNA exhibited albino phenotype in their eyes and skin (black arrows, \#4, \#16, \#17 and \#18). (F) Representative results of phenotypes of mice from Pdcd1 targeting. Western blot (WB) showing that knockout of Pdcd1 leads to a decrease in PD-1 protein of \#5, \#8, \#13, \#15 and \#17. (G) The percentage of different mutation types in pups. Black represents percentage of blastocysts harboring induced stop codons; Red represents percentage of blastocysts harboring unwanted mutations only; Blue represents percentage of wild type blastocysts. The number was indicated on the chart. $(H)$ Representative alignments of modified sequences from newborn pups (\#16 and \#18) using microinjection of BE3 mRNA and sgRNAs into one-stage embryos. The PAM sequences and substitutions are highlighted in blue and red, respectively; The expectedly edited codons are underlined. (I) Summary of the numbers of embryos used and mutants targeting the Tyr and Pdcd1 sites.

out of $10(50 \%)$ (\#1-3, \#7 and \#8) blastocysts carried induced stop codon for Pdcd1-sg1, Pdcd1-sg1+2 and Pdcd1-sg1+2+3, respectively (Fig. 1C). It is notable that no indel was observed among all tested blastocysts (Fig. S4A). Only one blastocyst (\#5) harbored unwanted mutations (W12C, A13T and V14M) for Pdcd1-sg1+2+3 (Figs. 1C and S4A).

Our further study with BE4max showed more outstanding editing efficiency. For Tyr, 4 out of 10 (40\%) blastocysts (\#1, \#4, \#6 and \#9) for Tyr-sg1 harbored i-stop mutation, while 8 out of $10(80 \%)$ blastocysts (\#3-10) harbored i-stop mutation for Tyr-sg1+2+7 (Figs. 1D and S3E). For Pdcd1, 2 out of 10 blastocysts (20\%) (\#3 and \#8), 7 out of 10 blastocysts $(70 \%)$ (\#2-5 and \#7-9) carried induced stop codon for Pdcd1-sg1 and Pdcd1-sg1+2+3, respectively (Figs. 1D and S3D). Only one embryo (\#6) harbored 13 bp deletion at Tyr locus. These results further demonstrated the universaltiy of multiple sgRNAs strategy to facilitate efficient i-stop generation in mouse embryos.
With those successes in vitro, we assess whether our multiple sgRNAs strategy could achieve complete gene disruption though i-stop conversion in vivo. Consistent with previous experimental conditions, BE3 mRNA (total $50 \mathrm{ng} / \mu \mathrm{L}$ ) and sgRNAs (Experiment 1: Tyr-sg1+2, Pdcd1-sg1+2+3, total $25 \mathrm{ng} / \mu \mathrm{L}$ ) were co-injected into one-cell embryos to target $T y r$ and $P d c d 1$ simultaneously, and a total of 9 pups (Founder \#614) were obtained (Fig. 1I). The results of editing frequencies in tail DNA showed, 5 out of $9(55.6 \%)$ mice were identified carrying genome modification at Tyr or Pdcd1 loci. Among these 5 mice, 4 mice (Founder \#8, \#9, \#13 and \#14) harbored i-stop conversion at Tyr or Pdcd1 loci and the last one (Founder \#11) harbored A13T conversion only (frequency of $28.6 \%$ ) for Pdcd1-sg3. Further analysis of these mice indicated that Founder \#8 and \#9 harbored i-stop conversion for Tyr-sg1 at the frequencies of $33 \%$. Meanwhile, 3 founder mice (\#8, \#13 and \#14) harbored i-stop conversion at Pdcd1 with the frequencies ranging from $28.6 \%$ to $50 \%$ (Fig. S4B).

To increase the efficiency of BE3-mediated i-stop, we tried new sgRNA combinations (Experiment 2: Tyr-sg1+2+7, Pdcd1-sg1+2+3). The results showed, 10 out of $11(90.9 \%)$ pups harbored genome modification (Figs. 11 and S4C). Among these 10 mice, 8 (Founder \#1, \#3, \#4, \#15-18 and \#20) and 6 (Founder \#5, \#15-19) mice harbored i-stop conversion at Tyr and Pdcd1, respectively, with the frequencies ranging from $25 \%$ to $100 \%$. It is worth notifying that, only 2 out of 20 pups (Founder \#5, \#9) harbored $5 \mathrm{bp}$ and $2 \mathrm{bp}$ deletion at Pdcd1 locus, respectively, indicating BE3 is much more precise than wild type Cas9 (Fig. S4C).

Although founder \#8 and \#9 both harbored W272stop conversion at Tyr locus, none of the nine newborns (Experiment 1) displayed the albino phenotype of white skin indicating incomplete gene disruption (Fig. 1E). For those pups from BE3 combined with Tyr-sg1+2+7 and Pdcd1-sg1+2+3, 8 out of 11 newborns (Founder \#1, \#2, \#4, \#5, \#15-18) displayed Tyr-deficient mice phenotype indicating the increased Tyr gene disruption efficiency mediated by multiple sgRNA i-stop strategy. Interestingly, all of the phenotypic mice show white skin over whole body instead of black-andwhite skin, the mosaic phenotype which displayed by mice harboring mosaic gene disruption of Tyr as previous reported (Zuo et al., 2017). These data further suggest multiple sgRNAs facilitated i-stop conversion and gene disruption, which allows phenotype analysis of founder animals.

To our knowledge, PD-1 protein is encoded by the Pdcd1 gene and highly expressed in thymus (Yue et al., 2014). To further analyze PD-1 disruption, thymus tissues were isolated by autopsy from 6 mice $(\# 5, \# 8, \# 13, \# 14, \# 15$ and $\# 17)$. The results showed, Founder \#5 and \#15 harbored W12stop and Q79Stop conversion at the frequencies of $61 \%$ and $100 \%$, respectively. Meanwhile, W12stop conversion for Pdcd1-sg2 and Q167stop conversion for Pdcd1-sg3 were observed in Founder \#17 at the frequencies of $90 \%$ and $100 \%$, respectively. Founder \#8 and \#13 harbored W12stop conversion at the frequencies of $49.5 \%$ and $50 \%$, respectively. Founder \#14 harbored Q167stop conversion at the 


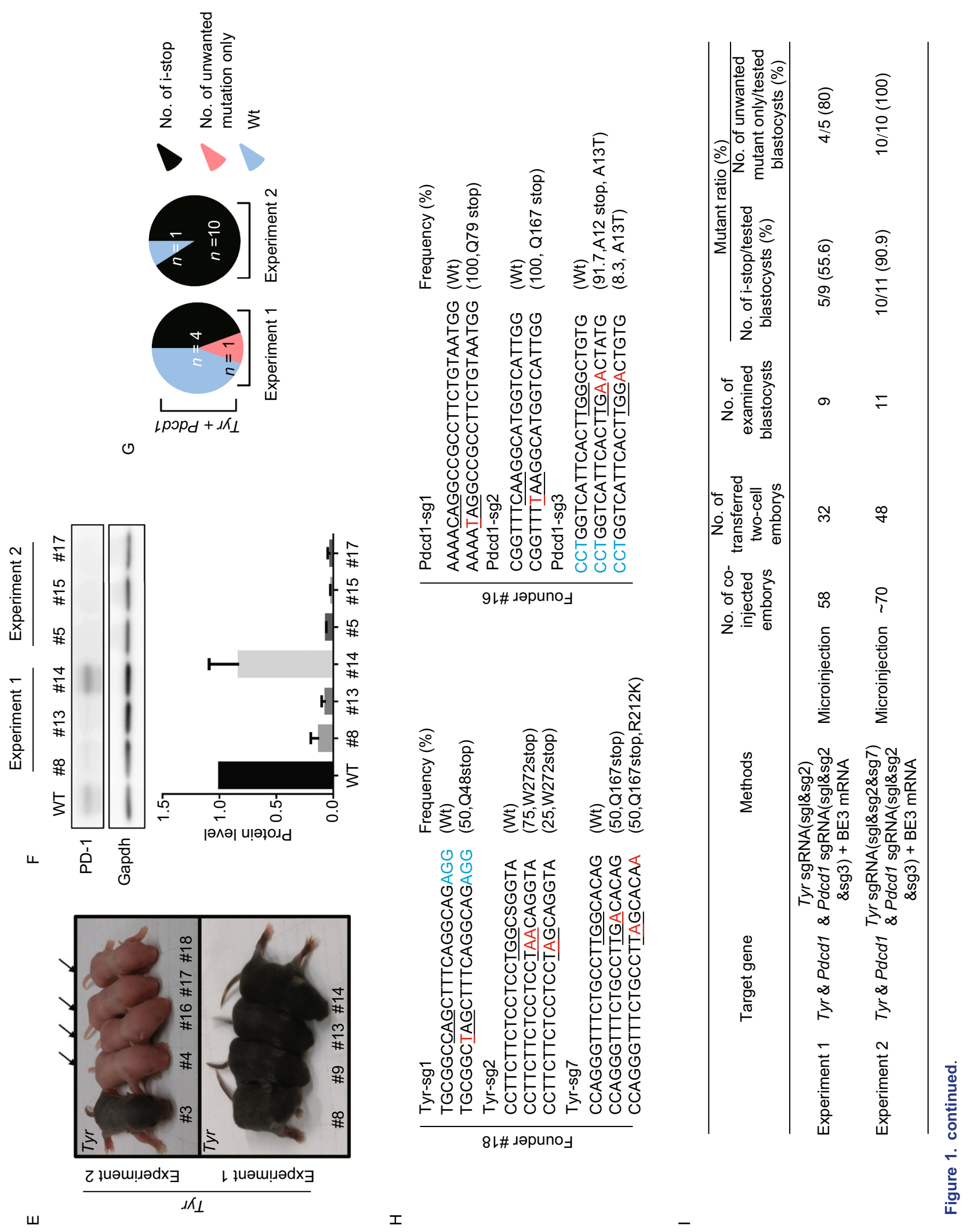


๘

$\infty$

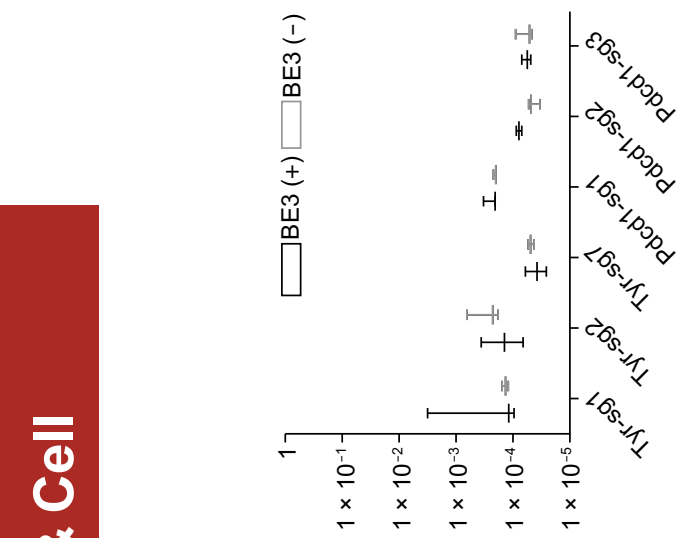

(\%) Кэuәnbəљу s|әрu|

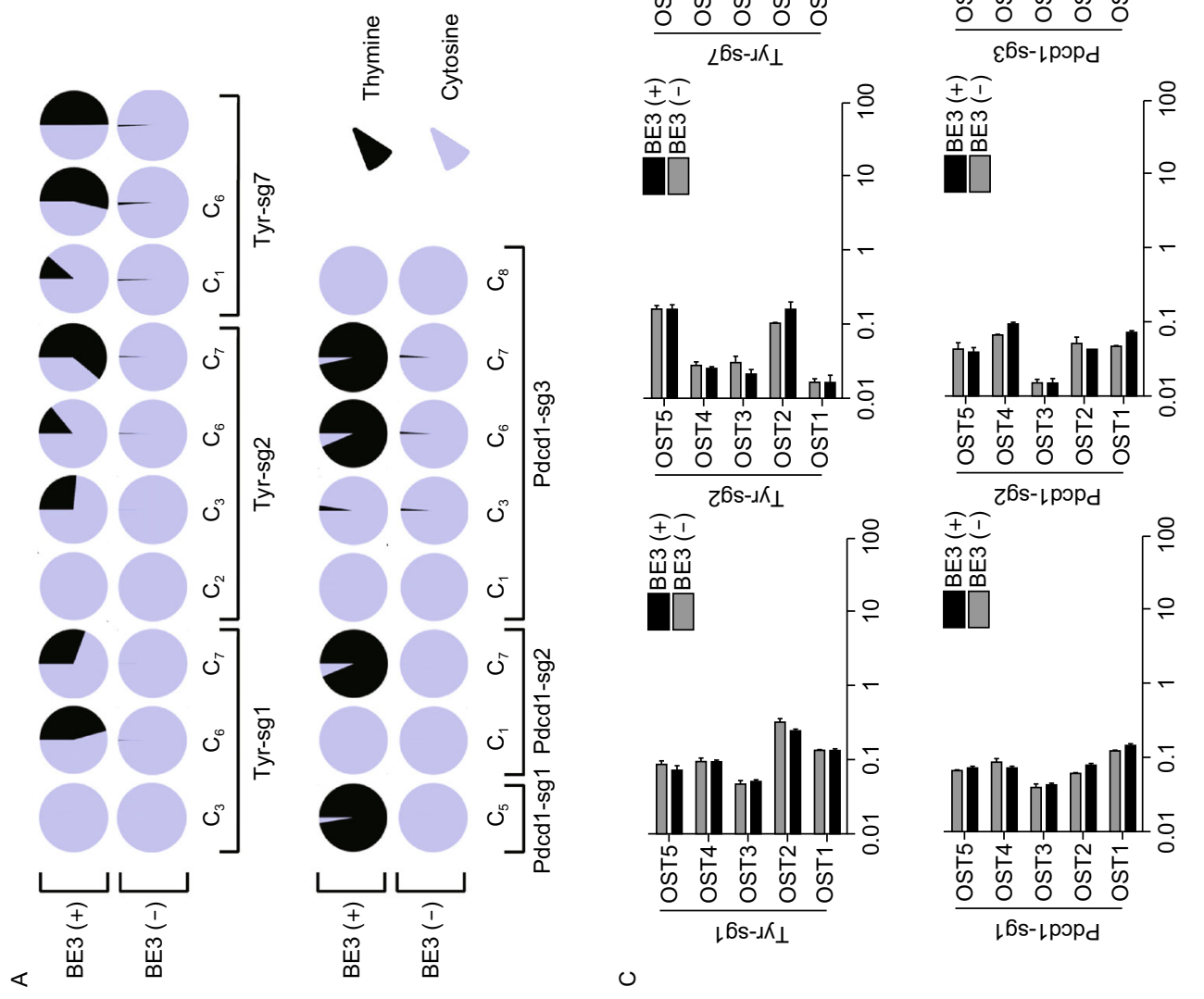

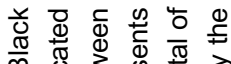

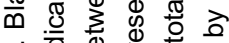
ठ்.

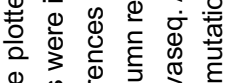

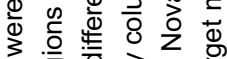
ब क्षे ब

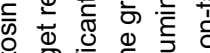

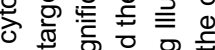
애 ब ह 응 㟧

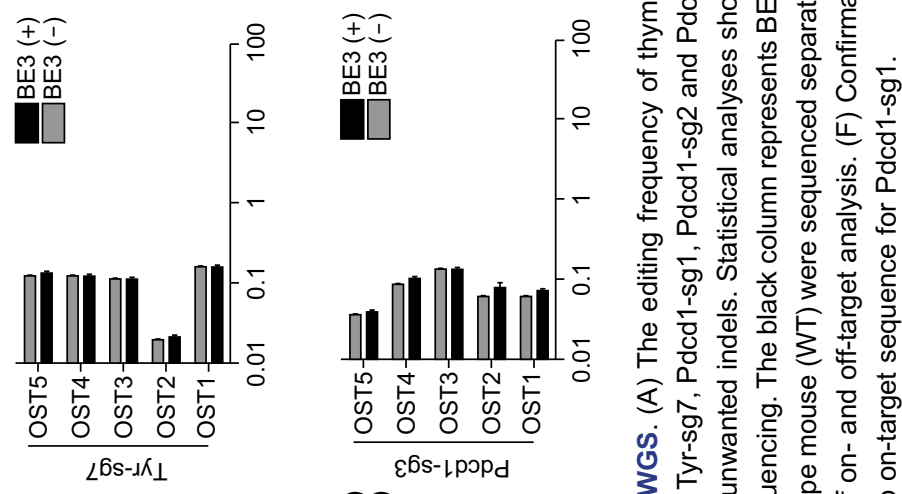

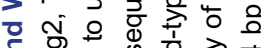

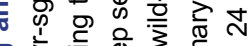

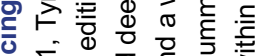

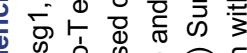

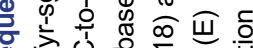

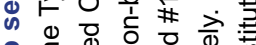

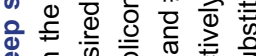

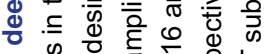
ठ

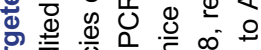

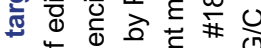
จ

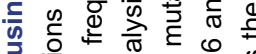
क力 층

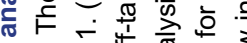
む)

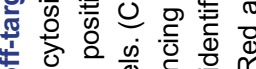
엉

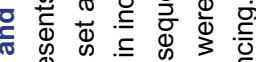

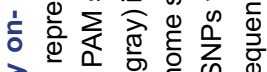
ते ते

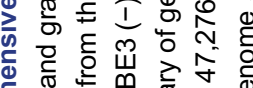

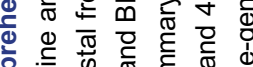

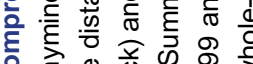
讨 娄 ن ن d 可产热出出 


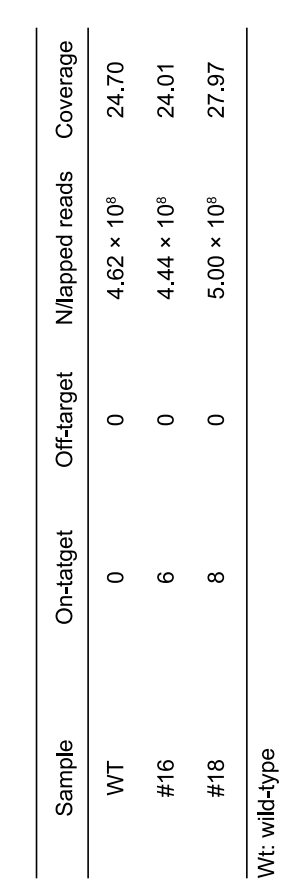

ш

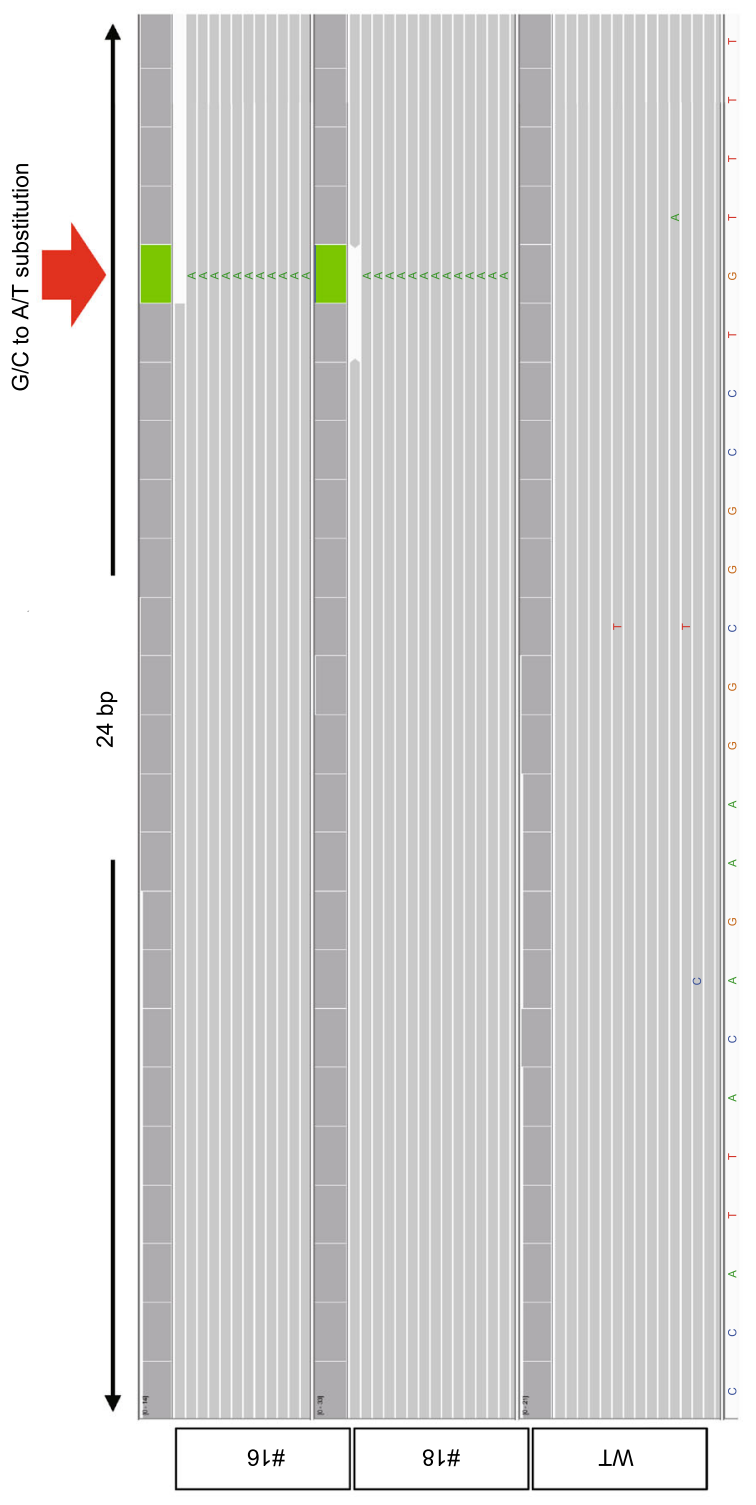

$\leftarrow$ $\frac{1}{0}$
$\frac{1}{\overline{0}}$
$\frac{0}{0}$
$\frac{0}{0}$

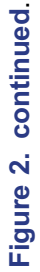


lower frequency of $30 \%$ (Fig. S4C). As expected, low PD-1 level was detected in $P d c d 1$ disruption mice $(\# 5, \# 8, \# 13$, \#15 and \#17). Among 6 tested mice, harboring high frequencies of i-stop were detected with significant reduction of PD-1 expression to $6 \%, 12.3 \%, 6.6 \%, 1.5 \%$ and $2.2 \%$, respectively (Fig. 1F). Founder \#14 harboring $30 \%$ Q167stop conversion was detected with normal PD-1 expression, which may be explained by the mosaicism. In addition, in total 20 newborns (9 plus 11), 5 mice (Founder $\# 8, \# 15, \# 16, \# 17$ and \#18) harbored i-stop conversion at both Tyr and Pdcd1, which demonstrated our strategy can simultaneously disrupt multiple genes in vivo.

We evaluated the on-/off-target effect though PCR-based deep sequencing. Using online tool (http://www.rgenome. net/cas-offinder/), we first selected 5 off-target sites for each sgRNA (Table S5). Off-target and on-target sites of six sgRNAs in this study were sequenced using tail DNA from four mice (\#4, \#16, \#17 and \#18), and 2 or 3 mice were analyzed for every sgRNA. Based on the sequencing results, no base substitution was detected at any off-target sites (30 sites in total) (Fig. 2C). To further explore the precision of BE3-mediated base editing, a WGS was performed using genomic DNA from two mutant mice (\#16 and \#18) and a wild-type mouse as the control at depth of about $24 \times$. We analyzed a total of 7,234 sites, including 1 on-target site and 1,069, 2,414, 1,919, 175, 961, 690 off-target sites (with up to 3-nucleatide mismatch) on Tyr-sg1, Tyr-sg2, Tyr-sg7, Pdcd1-sg1, Pdcd1-sg2 and Pdcd1-sg3, respectively (Figs. 2E and S5G). Only the C-to-T substitution within the target window was observed (Fig. 2F).

For on-target sites, the average conversions on targeted Cs were $45.75 \%\left(\mathrm{C}_{5}\right)$ and $30.63 \%\left(\mathrm{C}_{6}\right)$ for Tyr-sg1, 26.36\% $\left(\mathrm{C}_{3}\right), 14.06 \%\left(\mathrm{C}_{7}\right)$ and $61.03 \%\left(\mathrm{C}_{8}\right)$ for Tyr-sg2, and $15.05 \%$ $\left(C_{1}\right), 53.38 \%\left(C_{6}\right), 41.27 \%\left(C_{7}\right)$ for Tyr-sg3. Among these sgRNAs, only sgTyr-7 targeted $C_{1}$ locates out of the reported editing window $\left(\mathrm{C}_{4}-\mathrm{C}_{8}\right)$. Only one substitution was induced by Pdcd1-sg1 and Pdcd1-sg2 at $\mathrm{C}_{5}(97.44 \%)$ and $\mathrm{C}_{7}$ $(93.79 \%)$, respectively. Targeted substitutions at $\mathrm{C}_{3}, \mathrm{C}_{6}$ and $\mathrm{C}_{7}$ were observed at the frequencies of $2.48 \%, 93.67 \%$ and 96.55\% respectively for Pdcd1-sg3 (Fig. 2A). As expected, no detectable indel was observed at on-target sites (Fig. 2B). Taken together, multiple sgRNAs perform precise BE3 editing.

Continuous modifications are being made to base editor ever since its discovery to improve the efficiency or precision. From BE1 to current BE4, different components were engineered in base editor system, resulting in steady improvement of editing efficiency. Unlike those biostructural modification, a new strategy was utilized in our study to improve editing efficiency. In summary, we utilized multiple sgRNAs to facilitate i-stop generation of two endogenous genes mediated by different base editor (BE3 and BE4max), resulting in efficient and multiple gene disruption. High throughout sequencing analysis showed multiple sgRNAs strategy facilitated editing is precise with minimal off-target effect and indel. Although the concentration of injected materials was not test, typical concentrations of base editor $(50 \mathrm{ng} / \mu \mathrm{L})$ and sgRNA ( $25 \mathrm{ng} / \mu \mathrm{L})$ already achieved decent performance in zygote microinjection. Further studies could be carried out to titrate the injection concentrations and refine the protocol. Taken together, multiple sgRNAs is a universal strategy to achieve efficient gene knockout for phenotype analysis.

\section{FOOTNOTES}

The authors declare no Competing Financial or Non-Financial Interests. This work was supported by National Center for International Research (2017B01012), National Natural Science Foundation of China (Grant Nos. 31771299, 81600380 and 31600958), Natural Science Foundation of Jiangsu Province (BK20160313, BK20160317).

Kun Jia, Zongyang Lu, Fei Zhou, Zhiqi Xiong, Rui Zhang, Zhiwei Liu, Yu'e Ma, Lei He, Cong Li, Zhen Zhu, Dejing Pan, Zhengxing Lian declare that they have no conflict of interest. All institutional and national guidelines for the care and use of laboratory animals were followed.

Z.X.L. and D.P. conceived, designed and supervised the project. K.J., Z.Y.L. and F.Z. performed all experiments with the help of Z.X., R.Z. and Y.M.. K.J. and Z.Y.L. wrote the paper with inputs from Z.L., L.H., C.L., and Z.Z.. Z.X.L. managed the project.

Kun $\mathrm{Jia}^{1}$, Zongyang $\mathrm{Lu}^{3}$, Fei Zhou ${ }^{2}$, Zhiqi Xiong ${ }^{3}$, Rui Zhang ${ }^{1}$, Zhiwei $\mathrm{Liu}^{2}$, Yu'e $\mathrm{Ma}^{2}$, Lei He${ }^{2}$, Cong $\mathrm{Li}^{2}$, Zhen Zhu ${ }^{4}$, Dejing Pan ${ }^{2 凶}$, Zhengxing Lian ${ }^{1 凶}$

\footnotetext{
${ }^{1}$ Beijing Key Laboratory of Animal Genetic Improvement, China Agricultural University, Beijing 100094, China

2 Cambridge-Suda Genomic Resource Center and Jiangsu Key Laboratory of Neuropsychiatric Diseases Research, Soochow University, Suzhou 215123, China

3 School of Life Science and Technology, ShanghaiTech University, Shanghai 201210, China

${ }^{4}$ Key Laboratory of MEMS of Ministry of Education, Southeast University, Nanjing 210096, China

$\triangle$ Correspondence: pandejing@suda.edu.cn (D. Pan), lianzhx@cau.edu.cn (Z. Lian)
}

\section{OPEN ACCESS}

This article is distributed under the terms of the Creative Commons Attribution 4.0 International License (http://creativecommons.org/ licenses/by/4.0/), which permits unrestricted use, distribution, and reproduction in any medium, provided you give appropriate credit to the original author(s) and the source, provide a link to the Creative Commons license, and indicate if changes were made. 


\section{REFERENCES}

Cho SW, Kim S, Kim Y, Kweon J, Kim HS, Bae S, Kim JS (2014) Analysis of off-target effects of CRISPR/Cas-derived RNA-guided endonucleases and nickases. Genome Res 24:132-141

Fife BT, Pauken KE (2011) The role of the PD-1 pathway in autoimmunity and peripheral tolerance. Ann $N$ Y Acad Sci 1217:45-59

Hsu PD, Lander ES, Zhang F (2014) Development and applications of CRISPR-Cas9 for genome engineering. Cell 157:1262-1278

Hsu PD, Scott DA, Weinstein JA, Ran FA, Konermann S, Agarwala V, Li Y, Fine EJ, Wu X, Shalem O et al (2013) DNA targeting specificity of RNA-guided Cas9 nucleases. Nat Biotechnol 31:827-832

Kim YB, Komor AC, Levy JM, Packer MS, Zhao KT, Liu DR (2017) Increasing the genome-targeting scope and precision of base editing with engineered Cas9-cytidine deaminase fusions. Nat Biotechnol 35:371-376

Koblan LW, Doman JL, Wilson C, Levy JM, Tay T, Newby GA, Maianti JP, Raguram A, Liu DR (2018) Improving cytidine and adenine base editors by expression optimization and ancestral reconstruction. Nat Biotechnol 36:843-846

Komor AC, Kim YB, Packer MS, Zuris JA, Liu DR (2016) Programmable editing of a target base in genomic DNA without double-stranded DNA cleavage. Nature 533:420-424
Komor AC, Zhao KT, Packer MS, Gaudelli NM, Waterbury AL, Koblan LW, Kim YB, Badran AH, Liu DR (2017) Improved base excision repair inhibition and bacteriophage $\mathrm{Mu}$ Gam protein yields C:G-to-T: a base editors with higher efficiency and product purity. Sci Adv 3:eaao4774

Kuscu C, Parlak M, Tufan T, Yang J, Szlachta K, Wei X, Mammadov R, Adli M (2017) CRISPR-STOP: gene silencing through baseediting-induced nonsense mutations. Nat Methods 14:710-712

Pattanayak V, Lin S, Guilinger JP, Ma E, Doudna JA, Liu DR (2013) High-throughput profiling of off-target DNA cleavage reveals RNA-programmed Cas9 nuclease specificity. Nat Biotechnol 31:839-843

Witkop CJ (1979) Albinism: hematologic-storage disease, susceptibility to skin cancer, and optic neuronal defects shared in all types of oculocutaneous and ocular albinism. Ala J Med Sci 16:327-330

Yue F, Cheng Y, Breschi A, Vierstra J, Wu W, Ryba T, Sandstrom R, Ma Z, Davis C, Pope BD et al (2014) A comparative encyclopedia of DNA elements in the mouse genome. Nature 515:355-364

Zuo E, Cai YJ, Li K, Wei Y, Wang BA, Sun Y, Liu Z, Liu J, Hu X, Wei $W$ et al (2017) One-step generation of complete gene knockout mice and monkeys by CRISPR/Cas9-mediated gene editing with multiple sgRNAs. Cell Res 27:933-945
Kun Jia, Zongyang Lu and Fei Zhou have contributed equally to this work

Electronic supplementary material The online version of this article (https://doi.org/10.1007/s13238-019-0611-6) contains supplementary material, which is available to authorized users. 